example of the effect produced upon a borly by placing it in a magnetic field.

Now a bar of iron when brought under the influence of an electro-magnet becomes itself a magnet, posiessing north and south poles and manifesting all the phenomena of magnetism. This manifestation of magnetism is generally believer to be due to a particular arrangement of the molecules of the bar of iron effected by the power of the magnet-an arrangement in which the axes and similar poles of the nolecules aro made to point in the same direction. In the case of steel it is thought that the molecules, after being once arranged with their poles all pointing north and south, retain that position owing to the material condition of the bar. Consequently, when soft iron, steel, or, indeed, any form of matter-magnetic or diamagnetic-is subjected to the influence of a magnet, the power' of the magnet so far is of little consequence, and the only result that can occur as a direct effect of the magnetism is a change in the direction of the molecules, determined by their own in- dividual character and in accordance with the force which is acting upon them. But, further, it is probable that the diamagnetic molecules in the living body are continuously rotating upon a definite axis, the axis of rotation in every case being determined by the relation which each form of matter has to the magnetic force. I venture to hold this - opinion because a sphere of copper (which is one of the most diamagnetic forms of matter) will rotate upon its axis when suspended in a magnetic field ; ${ }^{2}$ and $I$ have myself ascertained from experiment that the clirection of the rotation depends upon the position of the magnetic poles in relation to the sphere, whilst the rapidity of the rotation is in proportion to the intensity of the magnetic field. From this fact in the case of copper I think we may not unjustly infer that other forms of diamagnetic matter, if subjected to the same conditions, would behave in a similar way; and if so, I think that we have in this fact a cause for those rotations under which inanimate matter is converted into living cells in the animal body through centripetal and centrifugal force, as described by Dr. Lionel Beale, ${ }^{3}$ whilst the selective influence which each form of matter, through its specific constitution and magnetic capacity, exercises would determine the character of the matter which each molecular germ withdraws from the nutrient plasma flowing over it. The character of the matter abstracted being thus cletermined by the polarity of the matter composing the germ, the importation of other matter of any kind into the blood corpuscles or into any tissue of the body, which is inconsistent with perfect health, would be productive of modifications of motion and of function, the natural tendency of which would be to produce disorder, disease, and death. In fact, this theory says that all disorder or disease is as much the outcome of the introduction of material particles into the system which should be foreign to it as the natural body is the result of the particular mode in which certain forms of matter uncter the influence of an all-pervading force are converter into it. I cannot, therefore, perceive that there would be any modification of natural phenomena under the conclitions to which you refer, other than those which are consistent with the main. tenance of the phenomena of life in a state of perfect health. In fact, is is clear that, under the known relations which exist between matter and the magnetic force, the only influence which magnetism can exercise upon the living body is to render the polarity of every atom engaged in the maintenance of life more marked, and therefore more capable of maintaining those phenomena which are naturally evolved during life by the material elements. And if this be so, there is no reason why an animal or person placed in the field of a strong electro-magnet should be conscious of the changed conditions; for as we are not conscions of natural and healthy bodily processes, such as the passage of the blood through the arteries and veins, the digestion of food, and the formation of tissue, so are we conscious of influence from an external source, snch as the electro-magnet in question, only to the extent to which natural functions of the body are affected or modified thereby. But when the body is subjected to the action of the electric force a totally clifferent condition of things exists, and the resulting phenomena must vary with the extent of the shock, whether it be in the form of static or dynamic charge. And this leads me on to reply

2 I exhibited this experiment at a conversazione of the Royal Society in May, 1887, and subsequently before the Physical Society of London. third edition, p. $277, \& c$. vely briefly to the note of Dr. McClure which appeared in the last issue of I'IL L L ANCE'T.

In the first place I would remark that in discussing the nature of the conductive or the dielectric properties of matter a clue recognition of the broar principle that all bories are conductors of electricity will tend to render this rather abstruse subject somewhat more easy of comprehension. It is a fact that every form of matter has its own specific power of conducting a current; and some bodies transmit the force with such great resistance that Faraday gave them the name of clielectrics, for they appear to convey the force from molecule to molecule more by induction than by conduction. In these substances, therefore, in proportion to the greater resistance which they oppose to the passage of the current, the presence and force of the electric energy will be most clearly manifested by the production of heat and disruptive effects. In the animal body the blood is unquestionably the best conductor-i.e., it is the substance which most strongly attracts the charge of electricity and transmits it to the earth. In this respect it performs the function of an ordinary lightning conductor. But the other tissues, in proportion to their resistance, may be classified as dielectrics, for they tend to throw the energy outwards more or less at right angles to the direction of the current, and they are in such a degree liable to suffer from disruptive effects. Whilst thanking Dr. McClure for his criticism of my paper, I would add that I quite concur with him in his remark that in the proper understanding of these phenomena lies the solution of many physiological problems. But I go further than this, and express my conviction that this magneto-electric theory of life involves the principle by which almost all vital phenomena may be explained.

Reiding, Sept. 21st, 1895 I am, Sirs, yours faithfully,

\section{"DIPHTHERIA IN LONDON."}

\author{
To the Editors of THE LINCET.
}

Sins, - I should like to say a word with reference to the annotation under the above heading which appeared in The LAXCET of Sept. 21st. During some investigations into an outbreak of scarlet ferer I came upon some conditions and usages of ordinary elementary school life which appeared to me to be likely to hare a distinct, and apparently previously unrecognised, influence on the spread of such diseases as scarlet fever and diphtheria. I found that the slates and pencils were used by the children quite indiscriminately, and that when it was necessary to rub out sums, copies, \&c. it was done by licking with the tongue and using saliva; and, moreover, that the slates were never washed. Taking into consideration the fact that the nasopharyngeal secretion in scarlet fever and diphtheria carries infection long after the patient is apparently convalescent, it is easy to understand that the circumstances abore mentioned would have a potent influence in spreading these diseases. I am, Sirs, yours faithfully,

Halesowen, Sept. 21st, 1895 .

T. BRETT-YOUNG.

\section{"THE MANCHESTER MEDICAL CRICKET CLUB."}

To the Editors of THE LANCET.

SrRs,-There is a slight inaccuracy in the article on the above subject in the current number of THE LANCET. This has been the fourth season since the club was constituted, and for two seasons previously a few matches were played, which led to the formation of the club.

I am, Sirs, yours faithfully, G. H. BroADBENT.

Ardwick-green, Manchester, Sept. 21st, 1895.

\section{"THE GRIEVANCES OF THE TELEGRAPH SERVICE CLERKS." \\ To the Editors of THE LANCET.}

SIRs, - I have no desire to extend any further what would doubtless be an unprofitable controversy concerning the authority of the figures I have drawn from the United Kingdom Postal and Telegraph Service Benevolent Society's reports with reference to the health and mortality of telegraph clerks. Suttice it to say that after thirteen years 